\title{
CHK2 is involved in the p53-independent radiosensitizing effects of valproic acid
}

\author{
DONG WAN $\mathrm{CHOO}^{1 *}$, SUNG HO GOH ${ }^{2 *}$, YOUNG WOO $\mathrm{CHO}^{1,3^{*}}$, HYE JUNG BAEK ${ }^{1}$, \\ EUN JUNG PARK ${ }^{4}$, NOBORU MOTOYAMA ${ }^{5,6}$, TAE HYUN KIM ${ }^{1}$, \\ JOO YOUNG KIM ${ }^{1}$ and SANG SOO KIM ${ }^{1}$
}

\author{
${ }^{1}$ Radiation Medicine Branch and ${ }^{2}$ Cancer Genomics Branch, National Cancer Center, Goyang, \\ Gyeonggi 410-769, Republic of Korea; ${ }^{3}$ Colgate University, Hamilton, NY 13346, USA; ${ }^{4}$ Cancer Immunology Branch, \\ National Cancer Center, Goyang, Gyeonggi 410-769, Republic of Korea; ${ }^{5}$ Department of Cognitive Brain Science, \\ National Centre for Geriatrics and Gerontology, Obu, Aichi 474-8522; ${ }^{6}$ Department of Aging Research, \\ Nagoya University Graduate School of Medicine, Nagoya, Aichi 466-8550, Japan
}

Received April 25, 2016; Accepted December 20, 2016

DOI: $10.3892 / 01.2017 .5792$

\begin{abstract}
Radiotherapy is an effective treatment for the majority of types of localized solid cancer. However, the risk of side effects to the surrounding normal tissues limits radiotherapeutic approaches. Whilst the mechanism of action of valproic acid, an inhibitor of histone deacetylase, remains unknown, the inhibitor is a potential antineoplastic radiosensitizer. The present study demonstrated the in vitro radiosensitizing effects of valproic acid on the human breast cancer MCF7 cell line, and revealed that valproic acid increased the level of DNA breakage, apoptosis and senescence. In addition, western blot analyses revealed that valproic acid induced tumor suppressor protein (p)53 and p21 expression, and activated checkpoint kinase 2 (CHK2) in MCF7 cells and primary mouse embryonic fibroblasts. Notably, treatment with valproic acid also induced increases in the level of p21 protein levels and CHK2 activity in p53-null colon cancer HCT116 cells. Furthermore, the present study demonstrated that valproic acid-induced radiosensitization was largely dependent on the activity of CHK2. The results of the present study reveal that valproic acid may exhibit clinical utility with respect to increasing the anticancer efficacy of radiotherapy by affecting the level of p53.
\end{abstract}

Correspondence to: Dr Sang Soo Kim, Radiation Medicine Branch, National Cancer Center, 323 Ilsan-ro, Ilsandong-gu, Goyang, Gyeonggi 410-769, Republic of Korea

E-mail: sangsookim@ncc.re.kr

${ }^{*}$ Contributed equally

Key words: valproic acid, checkpoint kinase 2, p21, senescence, radiosensitizer

\section{Introduction}

Epigenetic modifications of chromatin, such as acetylation, methylation and the phosphorylation of histones, result in dynamic changes to the structure of chromatin in a diverse range of biological conditions, which affect the transcriptional activity of the associated genes including those in the SWItch/Sucrose Non-Fermentable subfamily by altering chromatin packaging (1). The accumulation of acetylated histones, catalyzed by histone acetyltransferase (HAT), neutralizes positively charged lysines, which alters the conformation of chromatin and provides greater access to the promoter regions of the genes for the transcription factor complexes (2). Conversely, histone deacetylase (HDAC) removes the acetyl group from the lysine residues, leading to a condensation of chromatin and a transcriptional inactivation of the genes (3). There have been efforts to develop HDAC inhibitors as cancer or adjunct treatments. The exact mechanisms of action of these compounds remain unknown, but it has been hypothesized that the disturbance of proper gene expression by epigenetic pathways is involved $(3,4)$.

Valproic acid is a chemical compound that has been used clinically as an anticonvulsant and mood-stabilizing drug. However, valproic acid has also been demonstrated to inhibit HDAC enzymatic activity and enhance tumor cell radiotoxicity following irradiation (5-7). In addition to exhibiting an advantageous pharmacological profile, including oral bioavailability and minimal toxicity of the compound, valproic acid is considered to be a strong candidate for clinical use as a radiosensitizer.

The present study aimed to determine the anti-neoplastic potential of valproic acid combined with irradiation, demonstrating that the HDAC inhibitor conferred radiosensitivity, which caused DNA damage and decreased the rate of survival of cancer cells. Furthermore, valproic acid led to a marked accumulation of tumor suppressor protein (p) 21 in cells lacking p53, suggesting that valproic acid may be used in the treatment of various types of cancer with altered p53 status. Additionally, 
valproic acid-induced radiosensitization was associated with the activation of checkpoint kinase 2 (CHK2), suggesting an induction of a DNA-damage response. Collectively, these data suggest that the use of valproic acid as an adjuvant therapy provides a novel strategy for maximizing the therapeutic effectiveness of cancer radiotherapy.

\section{Materials and methods}

Cell culture and analysis. The MCF7 cells were obtained from the American Type Culture Collection (Manassas, VA, USA). To generate the mouse embryonic fibroblasts (MEFs), timed harem matings were produced by housing 4 female mice with one male/cage overnight and examining for vaginal plugs the following morning [0.5 days post coitum $(\mathrm{dpc})=$ noon of the day when a vaginal plug was found]. MEFs were obtained from embryos at $14.5 \mathrm{dpc}$ as described previously (8). p53- and CHK2-mutant MEFs were generated from intercrosses between $\mathrm{p} 53^{+-}$and $\mathrm{Chk} 2^{+/-}$mice, respectively, according to previous protocol, and did not exhibit any detectable phenotype until breeding $(9,10)$. All animal procedures were approved by the Institutional Animal Care and Use Committee of the National Cancer Center, in accordance with the National Institutes of Health Guide for the Care and Use of Animals. All comparisons between wild-type and mutant MEFs were performed within the same littermate-derived cells. The $150 \mathrm{mM}$ valproic acid stock solution was prepared in absolute ethanol and consequently diluted into $20 \%$ fetal bovine serum containing Dulbecco's modified Eagle medium prior to treatment. The cells were pretreated with the indicated concentrations of valproic acid 1 day prior to irradiation, then harvested for additional analysis.

Colony-formation assays were performed as previously described (11). Trypsin-harvested cells were counted using a Z1 Coulter Counter (Beckman Coulter, Inc., Brea, CA, USA), and were plated at a density of 200 cells/ $60-\mathrm{mm}$ dish. Subsequent to a 2 -week culture, the colonies were fixed in methanol and stained with Giemsa solution (Fluka, Sigma-Aldrich; Merck Millipore, Darmstadt, Germany). The number of colonies for each experimental condition was determined by averaging the results from triplicate plates. MTT assays were performed using an in vitro toxicology assay kit (Sigma-Aldrich; Merck Millipore) according to the manufacturer's protocol. Senescence staining was performed using a senescence $\beta$-galactosidase staining kit (cat. no. 9860; Cell Signaling Technology, Inc., Danvers, MA, USA) according to the manufacturer's protocol. In brief, $2 \%$ formaldehyde and $0.2 \%$ glutaraldehyde-fixed cells were incubated at $37^{\circ} \mathrm{C}$ with senescence staining solution, consisting of the following: $1 \mathrm{mg} / \mathrm{ml}$ of 5-bromo-4-chloro-3-indolyl P3-D-galactoside (X-Gal); $40 \mathrm{mM}$ citric acid; sodium phosphate, $\mathrm{pH}$ 6.0; $5 \mathrm{mM}$ potassium ferrocyanide; $150 \mathrm{mM} \mathrm{NaCl} ; 2 \mathrm{mM} \mathrm{MgCl}_{2}$.

For the flow cytometry analyses, the cells were harvested using trypsinization, fixed in $70 \%$ ethanol, and resuspended in propidium iodide solution. Cellular fluorescence was monitored using a FACSCalibur flow cytometer (BD Biosciences, Franklin Lakes, NJ, USA). DNA content and cell-cycle distribution were analyzed using CellQuest (version 5.1.2; BD Biosciences) and ModFit LT version 3.0 (Verity Software House, Topsham, ME, USA) software.
Comet assay. All comet assays were carried out under denatured conditions using a Comet assay kit (Trevigen, Inc., Gaithersburg, MD, USA), according to manufacturer's protocol. The MCF7 cells treated with valproic acid and/or irradiation were harvested using trypsinization, mixed with low-melting agarose at $37^{\circ} \mathrm{C}$ and plated onto comet slides. The slides were immersed in a pre-chilled lysis solution and incubated at $4^{\circ} \mathrm{C}$ overnight, and then electrophoresed and stained in $2.5 \mu \mathrm{g} / \mathrm{ml}$ propidium iodide solution. The lengths of the comets were measured and analyzed using a Komet 4.0.2 image analysis system (Andor Technology, Ltd., Belfast, UK).

Western blot analysis. Western blot analyses were carried out using enhanced chemiluminescence detection, as previously described (12). Primary antibodies against the following proteins were used: p53 (cat. no. SC-126 for human p53; Santa Cruz Biotechnology, Inc., Dallas, TX, USA and cat. no. CM5 for mouse p53; Novocastra, Leica Biosystems GmbH, Wetzlar, Germany); phospho-ATM-Ser1981 (cat. no. 5883), phospho-ATR-Ser1981 (cat. no. 2853) and phospho-CHK2-Thr68 (cat. no. 2661) (all Cell Signaling Technology, Inc.); CHK2 (cat. no. 99,420; BD Pharmingen, San Diego, CA, USA); decoy receptor 2 (DCR2; cat. no. ADI-AAP-371; Enzo Life Sciences, Farmingdale, NY, USA); and $\beta$-actin (cat. no. SC-47,778), p21 (cat. no. SC-6246) and $\alpha$-tubulin (cat. no. SC-8035) (both Santa Cruz Biotechnology, Inc.).

Bioinformatic analyses. Gene expression datasets for the valproic acid-treated cancer and embryonic cells were searched and retrieved from the National Center for Biological Information (NCBI) Gene Expression Omnibus (GEO) database (13). Among the resulting query hits, the GSE51952 data series for hepatocellular cancer HepG2 cells and GSE23958 series for E14 MEFs treated with $2 \mathrm{mM}$ valproic acid were chosen. Data files were loaded onto Expander software (version 7.1; Tel Aviv University, Tel-Aviv, Israel) (14). Expression data were normalized using quantile-normalization, and differential expression between negative control and valproic acid treated groups was analyzed using Student's $t$-test (false discovery rate, $\mathrm{P}<0.05$ ). The biological and functional features of significantly upregulated or downregulated genes were analyzed using the database for annotation, visualization and integrated discovery bioinformatics resources (15). The gene set enrichment of differentially expressed genes was analyzed using Expander software.

Statistical analysis. An unpaired Student's t-test was used to compare differences as specified in the text. $\mathrm{P}<0.05$ was considered to indicate a statistically significant difference.

\section{Results}

Valproic acid exerts a radiosensitizing effect in MCF7 cells. To test whether valproic acid confers a tumor cell-killing advantage in radiotherapy, the present study examined the radiosensitizing effect of $1 \mathrm{mM}$ valproic acid by determining the survival rate of irradiated MCF7 cells in the absence and presence of valproic acid using colony-formation assays. Pretreatment of MCF7 cells with valproic acid significantly 
A

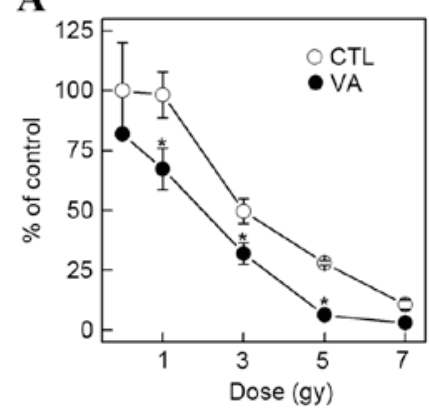

B

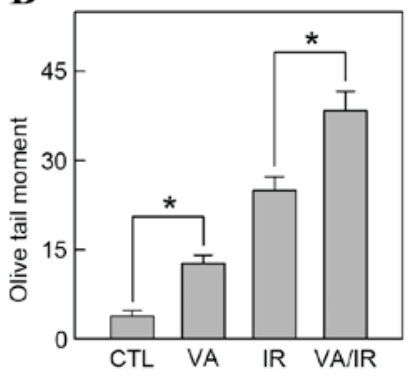

C

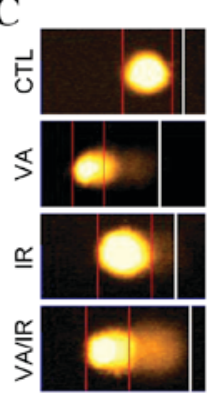

D

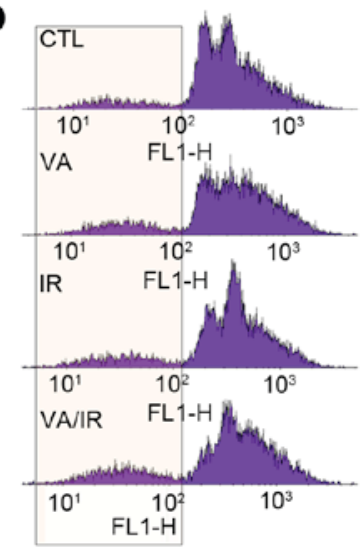

E

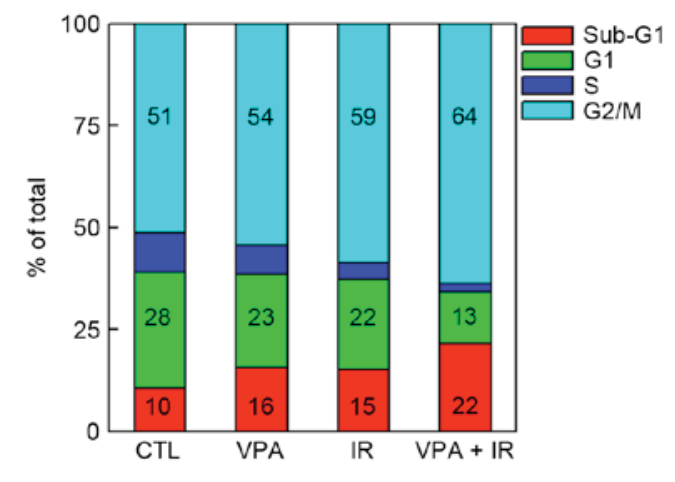

Figure 1. Valproic acid enhances the efficacy of radiation. (A) Colony-formation assays were performed using MCF7 cells treated with irradiation in the absence or presence of $1 \mathrm{mM}$ valproic acid. (B) Olive tail moments of the comet assay were calculated from at least 30 cells for each condition. (C) Representative images of MCF7 cells exposed to a $10 \mathrm{~Gy}$ dose of irradiation in the absence or presence of $2 \mathrm{mM}$ valproic acid were analyzed by comet assay under denaturing conditions. Each number represents the mean \pm standard error. (D) Flow cytometry detection of DNA content in MCF7 cells exposed to a 10 Gy dose of irradiation in the absence or presence of $2 \mathrm{mM}$ valproic acid. The boxed area indicates the apoptotic sub-G1 population. (E) \% cell distributions in the phases of the cell cycle subsequent to treatment. ${ }^{*} \mathrm{P}<0.05$. CTL, control; VA, valproic acid; IR, irradiation; VA/IR, valproic acid combined with irradiation.

reduced MCF7 cell survival compared with cells exposed to radiation only, over a range of $1-5$ Gy irradiation doses (1 Gy, $\mathrm{P}=0.036 ; 3 \mathrm{~Gy}, \mathrm{P}=0.022 ; 5 \mathrm{~Gy}, \mathrm{P}=0.001)$. Only the highest dose, $7 \mathrm{~Gy}$, in the colony-formation assays was not potentiated by valproic acid, as demonstrated in Fig. 1A.

To examine whether the radiosensitizing effect of valproic acid was a marker of the extent of DNA damage, the DNA damage in the absence and presence of valproic acid and irradiation was measured. The MCF7 cells were irradiated at a dose of $10 \mathrm{~Gy}$, with or without $2 \mathrm{mM}$ valproic acid, and the DNA strand breaks were measured by single-cell gel electrophoresis, comet assays, under denaturing conditions. Notably, the analysis demonstrated that valproic acid treatment significantly increased the level of DNA strand breaks compared with untreated cells (presence of radiation, $\mathrm{P}=0.0001$; absence of radiation 0.002; Fig. $1 \mathrm{~B}$ and $\mathrm{C}$ ). To determine whether the valproic acid-induced DNA damage conferred an increase in the level of apoptosis, a cell-cycle analysis of the MCF7 cells treated with $2 \mathrm{mM}$ valproic acid and exposed to a $10 \mathrm{~Gy}$ dose of irradiation was performed. Flow cytometry analyses revealed that valproic acid exposure increased the population of cells in the apoptotic sub-G1 phase from 10 to $16 \%$ in the absence of irradiation, and from 15 to $22 \%$ in the presence of irradiation, as demonstrated in Fig. 1D and E. These results indicated that the radiosensitizing effect of valproic acid was associated with an increase in the level of DNA breakage and apoptosis, which led to a reduction in the cell survival rate.
Valproic acid promotes MCF7 cell senescence. Although colony-formation assays are considered the 'gold standard' for measuring cell survival, the estimation of the effects of valproic acid on cell survival using the approach of the present study revealed that colonies from valproic acid-treated cells were smaller in size, with less cell spreading compared with untreated cells, regardless of the level of irradiation status, as illustrated in Fig. 2A. This result suggested that treatment with valproic acid inhibited the proliferation of tumor cells during colony formation. To understand the contribution of senescence, characterized by a spontaneous decline in growth rate during cell culture, the association between the decrease in colony size and senescence, which is essential for cell survival, was investigated. It was revealed that the treatment of MEFs with valproic acid increased the activity of senescence-associated $\beta$-galactosidase (SA- $\beta$-gal), a characteristic marker of senescence, as measured by X-gal staining and demonstrated in Fig. 2B. In addition, valproic acid induced a concentration-dependent accumulation of the senescence marker DCR2 in MEFs, as illustrated in Fig. 2C. Thus, valproic acid may induce premature senescence, which suppresses the growth of MCF7 colonies. To compensate for the difference in colony size, MTT assays were performed, and compared with the results of the colony-formation assay, as demonstrated in Fig. 2D. These results revealed that the survival rate of 1 Gy-irradiated MCF7 cells was 98 and $88 \%$ in colony-formation and MTT assays, respectively. However, 


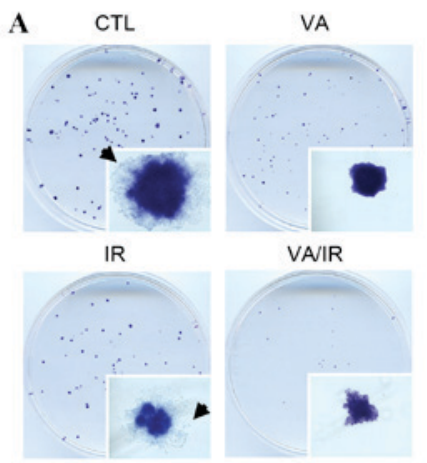

B

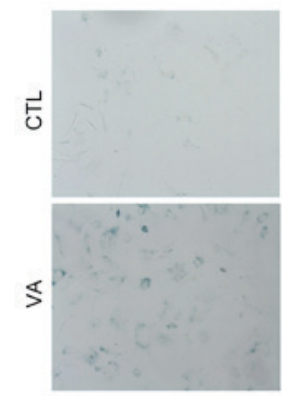

C

D

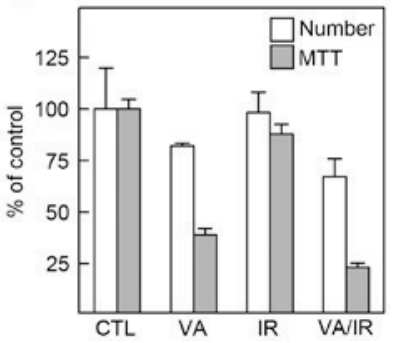

Figure 2. Valproic acid causes cellular senescence. (A) Colony morphologies are shown in MCF7 cells irradiated with a dose of $3 \mathrm{~Gy}$ in the absence or presence of $1 \mathrm{mM}$ valproic acid. Arrows in untreated and irradiated condition indicate colony spreading, which is deficient in colonies formed by valproic acid-treated cells. (B) Senescence phenotypes in valproic acid-treated MEFs $\mathrm{X}$-gal-stained, valproic acid-treated cells exhibited a flattened and enlarged morphology. (C) DcR2 protein levels were analyzed in MEFs treated with different concentrations of valproic acid, as indicated. $\alpha$-tubulin was used as a loading control. (D) MCF7 cell survival was measured by colony-formation and MTT assays following treatment with a dose of $1 \mathrm{~Gy}$ irradiation and/or $1 \mathrm{mM}$ valproic acid. CTL, control; VA, valproic acid; IR, irradiation; VA/IR, valproic acid combined with irradiation; DcR2, Decoy receptor 2; MEFs, mouse embryonic fibroblasts.

MCF7 cell survival following treatment with $1 \mathrm{mM}$ valproic acid was 82 and 39\% in colony formation and MTT assays, whereas the corresponding percentages for cells treated with valproic acid and irradiation were 67 and 23\%, respectively. These data indicated that valproic acid reduced the number of surviving cells, measured as colonies formed, and reduced the level of growth of MCF7 cells in surviving colonies, which efficiently prevents tumor growth.

Activation of CHK2 by valproic acid. To understand the association between valproic acid-induced cytotoxicity and DNA damage response, the levels of ATM serine/threonine kinase (ATM) and ataxia telangiectasia and Rad3-related protein (ATR) phosphorylation, initiators of DNA damage responses, were investigated in primary MEFs and MCF7 cells following treatment with valproic acid. The cells were treated with $1 \mathrm{mM}$ valproic acid for $24 \mathrm{~h}$, irradiated at a dose of $3 \mathrm{~Gy}$ and harvested at $1 \mathrm{~h}$ or 1 day following irradiation. As illustrated in Fig. 3A, irradiation immediately increased the levels of ATM phosphorylation. However, the levels of ATM and ATR phosphorylation were not affected by treatment with valproic acid, regardless of irradiation. The expression patterns of CHK2 and p53, downstream effectors of ATM, were examined under the same conditions. These experiments revealed an increase in the level of CHK2 in valproic acid-treated MEFs regardless of irradiation, as demonstrated in Fig. 3B. To confirm the contribution of valproic acid to CHK2 activation, the CHK2 status in MCF7 cells was examined. Western blot analysis revealed that the level of $\mathrm{CHK} 2$ phosphorylation in the valproic acid-treated MCF7 cells was higher compared with the untreated controls, regardless of irradiation, as illustrated in Fig. 3C. CHK2 accumulation was also greater in the MEFs treated with valproic acid and irradiated for up to 4 days, compared with the MEFs treated with irradiation only, as demonstrated in Fig. 3D. Thus, these data suggested that valproic acid is involved in the activation of $\mathrm{CHK} 2$, which enables the induction of a DNA damage response.

Valproic acid induces p21 expression in p53-null tumor cells. To assess whether the p53-dependent expression of p21 is required for the radiosensitizing effect of valproic acid, the wild-type p53 and p53-null colon cancer HCT116 cells were irradiated in the absence or presence of valproic acid, and the levels of p53 and p21 were investigated by western blot analysis. As demonstrated in Fig. 4A, irradiation of the wild-type p53-expressing HCT116 cells markedly increased p53 levels, which were also upregulated by treatment with valproic acid. The levels of p21 expression also increased subsequent to treatment with irradiation and valproic acid. Notably, treatment with valproic acid, but not irradiation, markedly induced the expression of p21 in the p53-null HCT116 cells. To confirm the p21-inducing effect of valproic acid in the absence of p53, the effect of valproic acid concentration and treatment duration on the expression patterns of CHK2, p53 and p21 in the p53 wild-type and p53-null primary MEFs were examined. The present study revealed that treatment with valproic acid induced p21 expression and CHK2 phosphorylation in the p53 $3^{-/}$and $\mathrm{p}^{2} 3^{+++}$MEFs, as demonstrated in Fig. 4B and C. In addition, longer incubations and higher concentrations of valproic acid resulted in the degeneration of the cytoskeletal network in the MEFs, although the $\mathrm{p} 53^{-/-}$MEFs were more resistant to this effect than the $\mathrm{p} 53^{+/+} \mathrm{MEFs}$. These data indicated that valproic acid induced the expression of p21 in the p53-null cell lines, suggesting that it may control the growth of p53-null tumors.

Prevention of CHK2 activation decreases valproic acid-induced growth suppression. To determine the contribution of CHK2 to valproic acid-induced p21 accumulation, the wild-type and CHK2-null MEFs were irradiated in the absence or presence of valproic acid, and the levels of p53 and p21 were investigated by western blot analysis. As demonstrated in Fig. 5A, irradiation induced p53 in the Chk $2^{+/+}$ MEFs, but not in the $\mathrm{Chk} 2^{-/-} \mathrm{MEFs}$. In addition, irradiation and treatment with valproic acid upregulated p21 in the $\mathrm{Chk}^{+/+}$ MEFs, but only irradiation induced an increase in p21 levels in the Chk2 $2^{-/-}$MEFs. To confirm the contribution of CHK2 to the valproic acid-induced radiosensitizing effect, the survival of the $\mathrm{Chk}^{+/+}$and $\mathrm{Chk} 2^{-/-}$MEFs in the absence or presence of valproic acid and irradiation was assessed, as illustrated in Fig. 5B. Treatment with irradiation and/or valproic acid reduced the survival of the $\mathrm{Chk} 2^{+/+}$MEFs by $>50 \%$, whereas the Chk2/- MEFs were significantly resistant (treatment with valproic acid, $\mathrm{P}=0.013$; irradiation, $\mathrm{P}=0.007$; combined treatment, $\mathrm{P}=0.016$ ) compared with the $\mathrm{Chk} 2^{+/+} \mathrm{MEFs}$ under every treatment condition. These results suggested that $\mathrm{CHK} 2$ is 
$\mathbf{A}$
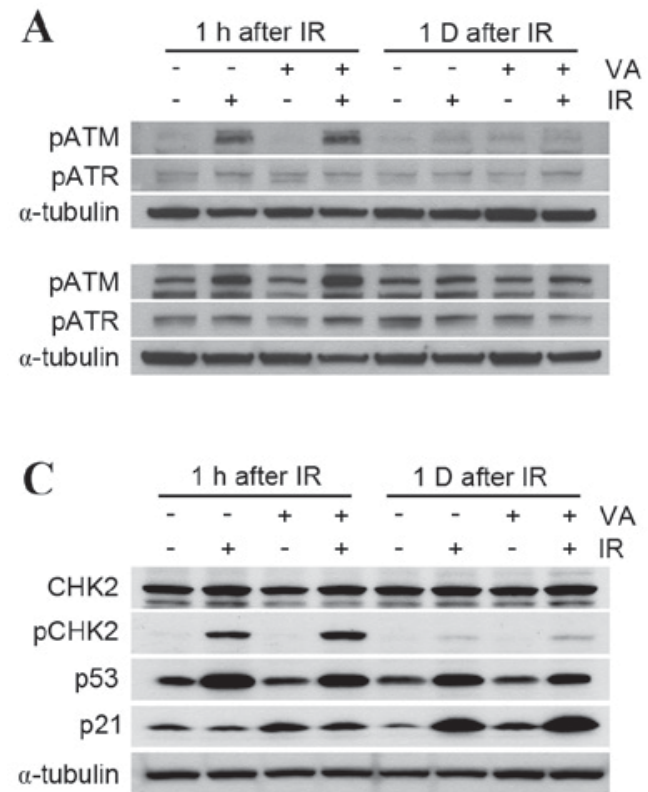
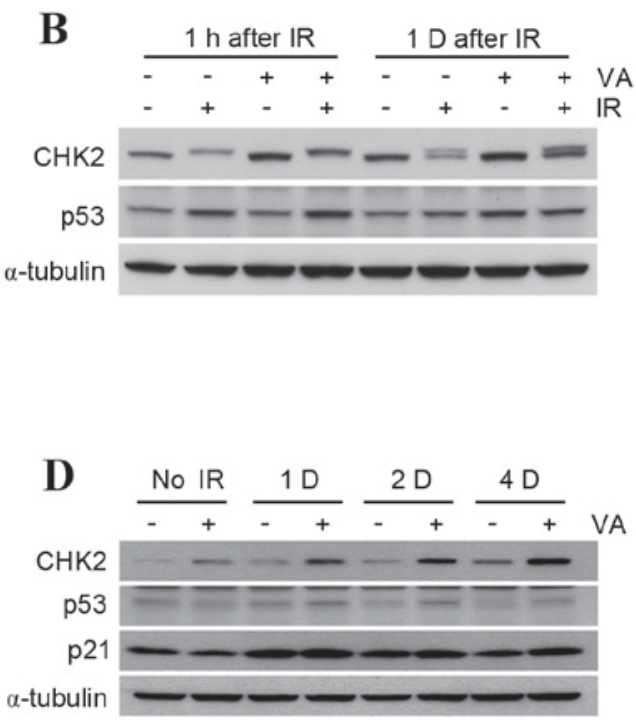

Figure 3. Valproic acid induces CHK2 activation. (A) Changes in level of phosphorylation of ATM and ATR subsequent to 10 Gy irradiation were analyzed in MCF7 cells (upper panel) and MEFs (lower panel) in the absence or presence of $1 \mathrm{mM}$ valproic acid pretreatment. (B) Levels of the DNA damage-response proteins CHK2, p53 and p21, and phosphorylated, or activated, CHK2 were measured in MEFs following treatment with valproic acid and irradiation. (C) The levels of DNA damage-response proteins, CHK2, p53 and p21, and phosphorylated, or activated, CHK2 were measured in MCF7 cells following treatment with valproic acid and irradiation. (D) Changes in DNA damage-response proteins in MEFs were analyzed by western blotting following treatment with valproic acid and irradiation. $\alpha$-tubulin was used as a loading control. MEFs, mouse embryonic fibroblasts; VA, valproic acid; IR, irradiation; D, day; ATM, ATM serine/threonine kinase; ATR, ataxia telangiectasia and Rad3-related protein; CHK2, checkpoint kinase 2; p53, tumor protein 53; p21, tumor protein 21.
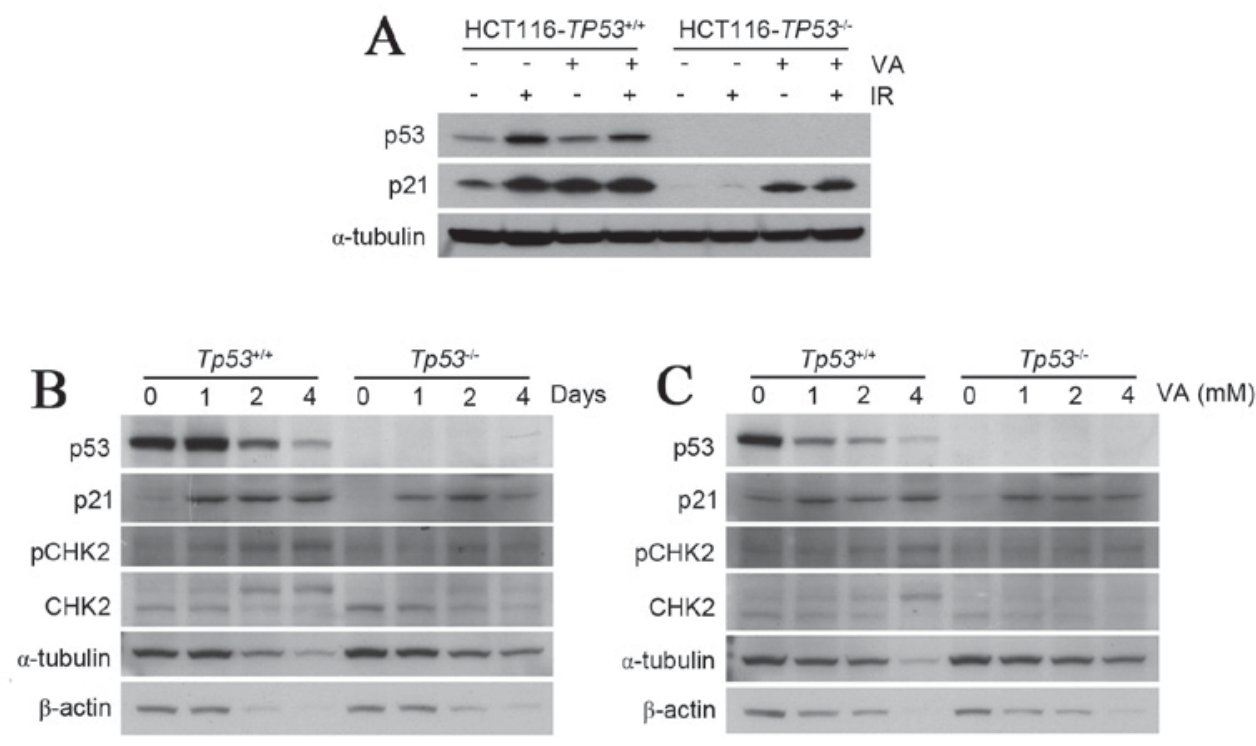

Figure 4. Valproic acid increases the level of p21 in p53-null cells. (A) The protein levels of p53 and p21 were measured in HCT116 (wild-type p53) and p53-null colon cancer HCT116 cells following treatment with valproic acid and irradiation. $\alpha$-tubulin was used as a loading control. (B) Changes in DNA damage-response proteins were analyzed by western blotting in $\mathrm{Tp} 53^{+/+}$and $\mathrm{Tp} 53^{-/}$MEFs following pretreatment with $1 \mathrm{mM}$ valproic acid for different times. (C) Changes in DNA damage-response proteins were analyzed by western blotting in $\mathrm{Tp} 53^{+/+}$and $\mathrm{Tp} 53^{-/}$MEFs following pretreatment with different concentrations of valproic acid for 1 day. $\beta$-actin and $\alpha$-tubulin were used as loading controls. p21, tumor protein $21 ; \mathrm{p} 53$, tumor protein 53 ; CHK2, checkpoint kinase 2; pCHK2, phosphorylated

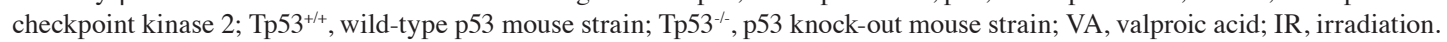

involved in the valproic acid-induced upregulation of p21 and suppression of cell growth.

Bioinformatic analyses of valproic acid-dependent differential gene expression. A bioinformatics analysis conducted using a similar data set (GSE51952) from the NCBI GEO public database revealed the differential expression of 924 genes following the treatment of $\mathrm{HepG} 2$ cells with $2 \mathrm{mM}$ valproic acid. The CHK2 transcript was not classified as the genes that showed significant differential expression, whereas the transcripts for p21 (CDKN1A), demonstrated in Fig. 6A, and CHK1, demonstrated in Fig. 6B, were significantly altered $(\mathrm{P}<0.0001)$. 

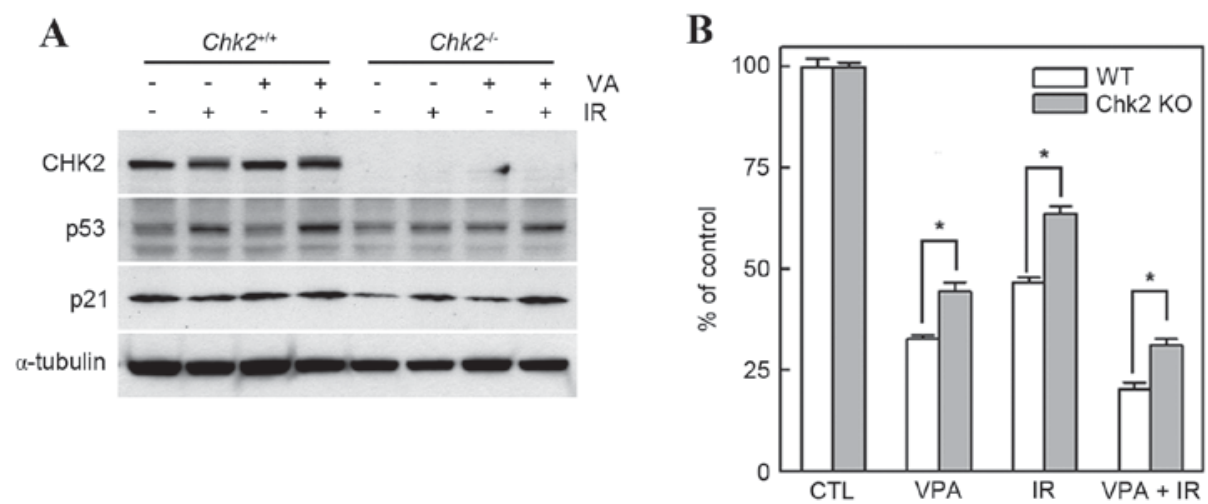

Figure 5. Loss of CHK2 reduces the radiosensitizing effect of valproic acid. (A) CHK2, p53, and p21 protein levels were measured in Chk $2^{++}$and Chk2 $2^{--} \mathrm{MEFs}$ following treatment with valproic acid and irradiation. $\alpha$-tubulin was used as a loading control. (B) The survival of Chk $2^{+/+}$and Chk $2^{-/}$MEFs following treatment with $1 \mathrm{mM}$ valproic acid and a $3 \mathrm{~Gy}$ dose of irradiation was measured by MTT assay. Each number represents the mean \pm standard error of quadruplicate determinations. ${ }^{~} \mathrm{P}<0.05$. CHK2, checkpoint kinase 2; Chk $2^{+/}$, checkpoint kinase 2 wild type mouse strain; Chk2 $2^{--}$, checkpoint kinase 2 knock-out mouse strain; p53, tumor protein 53; p21, tumor protein 21; CTL, control; VA, valproic acid; IR, irradiation; VA/IR, valproic acid combined with irradiation; KO, knockout; WT, wild type.

Notably, p21 was also part of a gene signature belonging to the 'response to toxin' or 'response to drug' categories of the Gene Ontology-Biological Process guidelines (16). In addition, the decrease in 553 expression in HepG2 cells suggested that the radiosensitizing effect of valproic acid was dependent on p53 expression and on the activities of other gene sets. The gene set enrichment analyses of differentially expressed genes subsequent to treatment with valproic acid demonstrated elevated enrichment scores for gene sets involved in 'G1 to S cell cycle control', 'TP53 network', 'miRNAs involved in DNA damage response' and 'statin pathway', as illustrated in Fig. 6C. The enrichment score at the peak indicates the degree to which a gene set is overrepresented at the top of a ranked list of genes, and the 'Hit', in the centre of the panel, indicates where the members of the gene set appear in the ranked list of genes (17). These results suggested that the significant increase in gene activities associated with the 'response to drug' or 'response to toxin' categories subsequent to treatment with valproic acid contributed to the susceptibility of cells to irradiation.

\section{Discussion}

The balance between chromatin condensation and decondensation, reflecting the nature and degree of histone modifications, is important for the regulation of transcription during cell survival and death. Thus, a dysregulation of HAT and HDAC activities is frequently associated with altered phenotypes, including cancer, highlighting the potential clinical utility of HDAC inhibitors as anticancer drugs. In addition to monotherapy trials, combined treatment with HDAC inhibitors and radiation is receiving attention due to the synergistic therapeutic effects demonstrated $(18,19)$. At present, diverse inhibitors of HDAC have been revealed to enhance radiosensitivity in vitro and in mouse xenograft models of human cell lines, although the detailed mechanisms responsible for this radiosensitizing effect remain unknown.

Valproic acid is a well-tolerated drug used to treat seizure disorders that has recently been demonstrated to inhibit HDAC (6). The results of the present study showed that valproic acid enhanced the radiosensitivity of human tumor cells and normal mouse cells. The in vitro experiments revealed that treatment with valproic acid suppressed MCF7 cell survival in colony-formation assays through interference with DNA breakage and apoptosis, analyzed by comet assays and fluorescence-activated cell sorting analysis, respectively. Treatment with valproic acid was revealed to reduce the levels of proliferation of cells in the surviving colonies compared with the untreated cells or the cells exposed to irradiation only. The normal MEFs cultured in valproic acid-containing media exhibited senescent morphology and expressed the cellular senescence marker, SA- $\beta$-gal. Subsequent western blot analyses demonstrated that valproic acid also altered the levels of the molecular marker of senescence, DCR2.

The combination of radiation and valproic acid revealed a greater ability of valproic acid to promote the accumulation of p53 for up to 4 days in wild-type MEFs compared with irradiation alone. A study using colorectal cancer HCT116 cells reported that valproic acid enhances tumor growth suppression subsequent to irradiation only in cells with a wild-type p53 status (20), suggesting that valproic acid-induced radiosensitization is p53-dependent. Valproic acid was subsequently suggested to stabilize a specific acetyl modification, lysine 120 , of the p53 tumor-suppressor protein, resulting in an increase in the proapoptotic function this protein at the mitochondrial membrane (21). The radiosensitizing effect of trichostatin $\mathrm{A}$, an additional HDAC inhibitor, has been suggested to be affected by the levels of p53 and ATM of the cells tested (22). However, in human cancer, $>50 \%$ of tumors contain a mutation or deletion of the p53 gene, which increases the likelihood for uncontrolled cell division (23), as p53 serves a central role in mediating the DNA-damage response through the transactivation of numerous growth-inhibitory or apoptotic genes, including p21 (24). Thus, the inactivation of p53 has been revealed to correlate with poor prognosis and resistance to chemotherapy and radiotherapy in malignant tumors $(25,26)$. Hence, establishing the dependence of the activities of anticancer drugs on the cellular expression of p53 is important. Notably, the results of the present study demonstrated that valproic acid increased the p21 levels in p53-null colon cancer HCT116 cells to a degree comparable to that of p53-wild type 

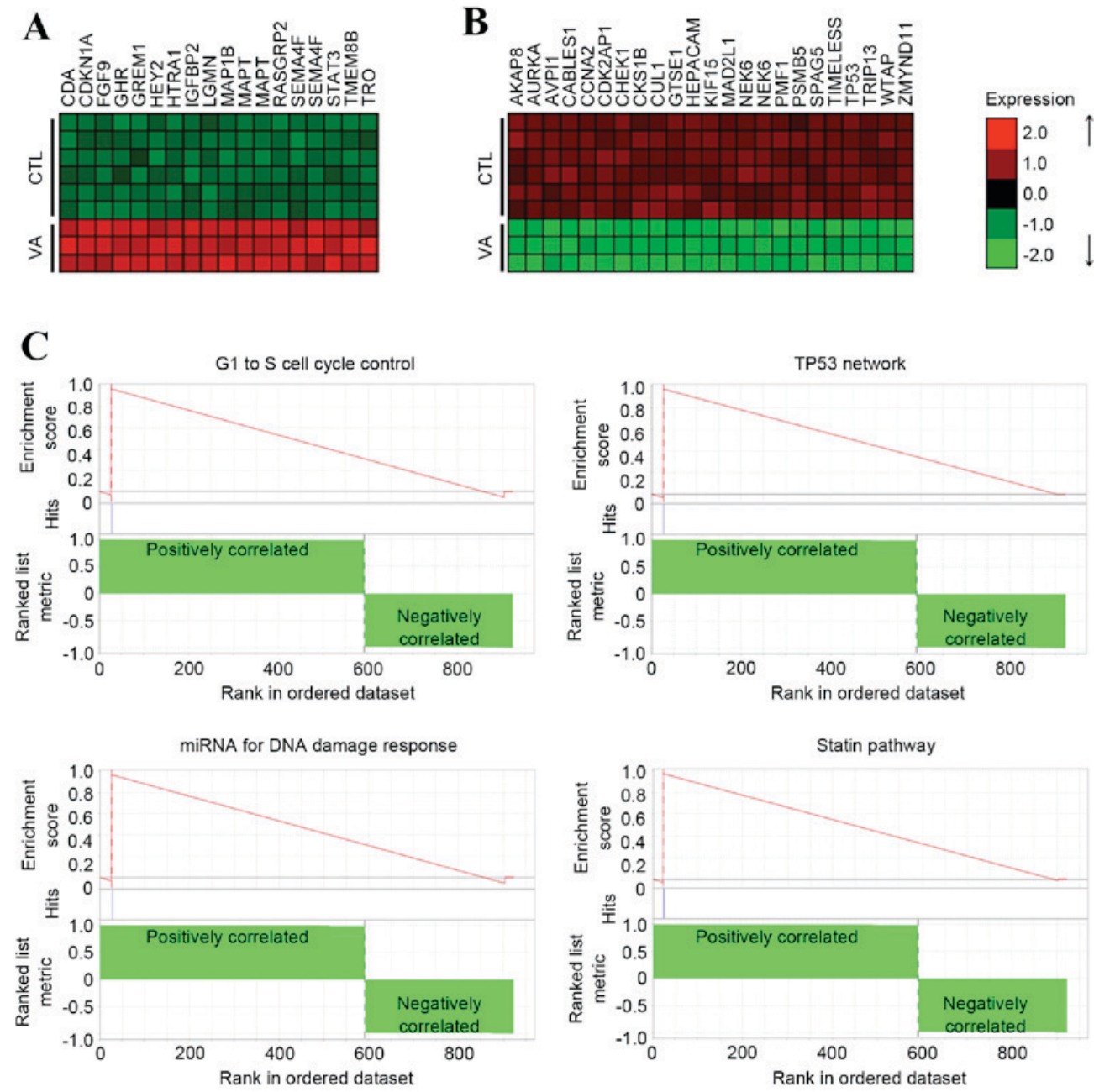

Figure 6. Differential gene expression profile of HepG2 cells following treatment with $2 \mathrm{mM}$ valproic acid for $24 \mathrm{~h}$. (A) Heatmap of upregulated gene expression signatures categorized into 'GO:00,40008 Regulation of growth' from analyses of 'Gene Ontology-Biological Process'. An increase in p21, or CDKN1A, expression was observed. (B) Heatmap of downregulated gene expression signatures categorized into 'GO:0,007049 Cell cycle' from analyses of 'Gene Ontology-Biological Process'. Genes associated with the cell cycle, including AURKA, CHEK1, CCNA2 and TP53 were downregulated following treatment with $2 \mathrm{mM}$ valproic acid. (C) Gene set enrichment analysis of 924 differentially expressed genes revealed highly enriched gene sets in HepG2 cells following treatment with $2 \mathrm{mM}$ valproic acid treatment for $24 \mathrm{~h}$. The significant gene sets ( $\mathrm{P}=0.014)$ were 'G1 to $\mathrm{S}$ cell cycle control', 'TP53 network', 'miRNA for DNA damage response' and the 'statin pathway'. The enrichment score at the peak reflects the degree to which a gene set is overrepresented at the top of a ranked list of genes. The middle portion, 'Hit', shows where the members of the gene set appear in the ranked list of genes. The ranking metric shows a phenotype of a positive correlation of 592 upregulated genes and a negative correlation of 332 downregulated genes with valproic acid treatment. miRNA, microRNA; TP53, tumor protein $\mathrm{p} 53$; VA, valproic acid; CTL, control.

cells, as illustrated in Fig. 4. As the inactivity of p53 in tumors is associated with resistance to a number of commonly used anticancer agents, valproic acid, which induced p21 independent of 553 , may be useful in the treatment of these types of tumors, independently or in combination with radiotherapy.

In the present study, it was also demonstrated that valproic acid activates CHK2, a kinase that phosphorylates p53 subsequent to DNA damage, in the MCF7 cells and primary MEFs. Valproic acid increased the levels of threonine- 68 phosphorylation of CHK2, and the protein levels of CHK2 in the MCF7 cells and primary MEFs, respectively. In the wild-type MEFs, treatment with valproic acid alone induced CHK2 activation, but the combined treatment with valproic acid and irradiation produced a greater effect. Prolonged treatment with valproic acid, lasting up to 3 days, in the presence of irradiation induced a greater degree of CHK2 expression in the primary MEFs, suggesting that the activation of CHK2 affects the radiosensitizing effect of valproic acid. A previous report using prostate cancer DU145 cells revealed that the combined pretreatment of valproic acid and 1,25-dihydroxyvitamin D3 enhanced the irradiation-induced activation of CHK2 by $39.0 \%$, compared with $23.8 \%$ in non-pretreated cells $(\mathrm{P}<0.05)(27)$. The treatment of cancer cells with valproic acid revealed a senescence-inducing activity and an induction of $\mathrm{p} 21$ expression, which correlated with the growth-inhibitory and antitumor activity of valproic acid $(28,29)$. Notably, senescence is associated with the phosphorylation and activation of CHK2 in human fibroblasts, and CHK2 inactivation in human fibroblasts has been shown to decrease the expression of p21 (30). In addition, the viral expression of $\mathrm{CHK} 2$ in p53-defective breast carcinoma SK-BR-3 cells and immortalized keratinocyte $\mathrm{HaCaT}$ cells leads to senescence and a transcriptional induction of $\mathrm{p} 21$, suggesting that a CHK2-mediated, p53-independent pathway may contribute to tumor suppression and affect the outcome of genotoxic treatments (31). The present study also confirmed that the deletion of CHK2 in the MEFs decreased the level of 
valproic acid-induced upregulation of p21 and suppression of cell growth, suggesting that CHK2 is involved in the radiosensitizing effects of valproic acid.

To identify the mechanisms responsible for the valproic acid-induced activation of CHK2, changes in the CHK2 transcript levels in the presence of valproic acid were investigated by reverse transcription quantitative polymerase chain reaction. However, this analysis revealed no significant induction of CHK2 transcripts (data not shown), suggesting that valproic acid modulated CHK2 levels and activity through a mechanism other than transcriptional control. Additional analyses of the bioinformatics data from HepG2 cells also revealed no changes in CHK2 transcripts, but did demonstrate a significant change in p21 and CHK1 transcripts. Notably, a pathway analysis of the valproic acid-treated cells showed a significant enrichment of gene sets for 'G1/S checkpoint', 'miRNAs involved in DNA damage response' and the 'statin pathway', in addition to 'Tp53 network'. These results suggested that the radiosensitizing effect of valproic acid is dependent on the status of p53, and the activity of other gene sets that have not yet been identified. Although the precise molecular mechanism by which valproic acid potentiates the effect of irradiation remains unknown, these data revealed that treatment with valproic acid and irradiation may enhance the radiosensitivity of p53-altered types of cancer.

\section{Acknowledgements}

The manuscript was edited by English Manager Science Editing (Coogee, Australia). The present study was supported by the grants from the National Cancer Center of Korea (grant no. NCC-1610030); the Korea Healthcare Technology R\&D Project (grant no. HI11C1289); and the National Research Foundation of Korea (grant no. 2014R1A2A1A11049935).

\section{References}

1. Huang C, Sloan EA and Boerkoel CF: Chromatin remodeling and human disease. Curr Opin Genet Dev 13: 246-252, 2003

2. Robertson KD: DNA methylation and chromatin-unraveling the tangled web. Oncogene 21: 5361-5379, 2002.

3. Carew JS, Giles FJ and Nawrocki ST: Histone deacetylase inhibitors: Mechanisms of cell death and promise in combination cancer therapy. Cancer Lett 269: 7-17, 2008.

4. Kristensen LS, Nielsen HM and Hansen LL: Epigenetics and cancer treatment. Eur J Pharmacol 625: 131-142, 2009.

5. Barker CA, Bishop AJ, Chang M, Beal K and Chan TA: Valproic acid use during radiation therapy for glioblastoma associated with improved survival. Int J Radiat Oncol Biol Phys 86: 504-509, 2013

6. Karagiannis TC, $\mathrm{Kn} \mathrm{H}$ and El-Osta A: The epigenetic modifier, valproic acid, enhances radiation sensitivity. Epigenetics 1: 131-137, 2006

7. Van Nifterik KA, Van den Berg J, Slotman BJ, Lafleur MV, Sminia P and Stalpers LJ: Valproic acid sensitizes human glioma cells for temozolomide and $\gamma$-radiation. J Neurooncol 107: 61-67, 2012.

8. Lim JA, Baek HJ, Jang MS, Choi EK, Lee YM, Lee SJ, Lim SC, Kim JY, Kim TH, Kim HS, et al: Loss of $\beta 2$-spectrin prevents cardiomyocyte differentiation and heart development. Cardiovase Res 101: 39-47, 2014.

9. Donehower LA, Harvey M, Slagle BL, McArthur MJ, Montgomery CA Jr, Butel JS and Bradley A: Mice deficient for p53 are developmentally normal but susceptible to spontaneous tumours. Nature 356: 215-221, 1992.

10. Takai H, Naka K, Okada Y, Watanabe M, Harada N, Saito S, Anderson CW, Appella E, Nakanishi M, Suzuki H, et al: Chk2-deficient mice exhibit radioresistance and defective p53-mediated transcription. EMBO J 21: 5195-5205, 2002.
11. Baek HJ, Kim TH, Shin D, Kwak JW, Choo DW, Lee SB, Furusawa Y, Ando K, Kim SS and Cho KH: Radiobiological characterization of proton beam at the National Cancer Center in Korea. J Radiat Res 49: 509-515, 2008.

12. Baek HJ, Lim SC, Kitisin K, Jogunoori W, Tang Y, Marshall MB, Mishra B, Kim TH, Cho KH, Kim SS and Mishra L: Hepatocellular cancer arises from loss of transforming growth factor beta signaling adaptor protein embryonic liver fodrin through abnormal angiogenesis. Hepatology 48: 1128-1137, 2008.

13. Barrett T, Wilhite SE, Ledoux P, Evangelista C, Kim IF, Tomashevsky M, Marshall KA, Phillippy KH, Sherman PM, Holko M, et al: NCBI GEO: Archive for functional genomics data sets-update. Nucleic Acids Res. 41 (Database Issue): D991-D995, 2013.

14. Shamir R, Maron-Katz A, Tanay A, Linhart C, Steinfeld I, Sharan R, Shiloh Y and Elkon R: EXPANDER-an integrative program suite for microarray data analysis. BMC Bioinformatics 6: 232, 2005.

15. Huang da W, Sherman BT and Lempicki RA: Systematic and integrative analysis of large gene lists using DAVID bioinformatics resources. Nat Protoc 4: 44-57, 2009.

16. The Gene Ontology Consortium: Gene ontology consortium: Going forward. Nucl Acids Res 43 (Database Issue): D1049-D1056, 2015.

17. Subramanian A, Tamayo P, Mootha VK, Mukherjee S, Ebert BL, Gillette MA, Paulovich A, Pomeroy SL, Golub TR, Lander ES and Mesirov JP: Gene set enrichment analysis: A knowledge-based approach for interpreting genome-wide expression profiles. Proc Natl Acad Sci USA 102: 15545-15550, 2005.

18. Cerna D, Camphausen K and Tofilon PJ: Histone deacetylation as a target for radiosensitization. Curr Top Dev Biol 73: 173-204, 2006.

19. De Schutter H and Nuyts S: Radiosensitizing potential of epigenetic anticancer drugs. Anticancer Agents Med Chem 9: 99-108, 2009.

20. Chen X, Wong P, Radany E and Wong JY: HDAC inhibitor, valproic acid, induces p53-dependent radiosensitization of colon cancer cells. Cancer Biother Radiopharm 24: 689-699, 2009.

21. Chen X, Wong JY, Wong P and Radany EH: Low-dose valproic acid enhances radiosensitivity of prostate cancer through acetylated p53-dependent modulation of mitochondrial membrane potential and apoptosis. Mol Cancer Res 9: 448-461, 2011.

22. Kim IA, Kim IH, Kim HJ, Chie EK and Kim JS: HDAC inhibitor-mediated radiosensitization in human carcinoma cells: A general phenomenon? J Radiat Res 51: 257-263, 2010.

23. Hollstein M, Sidransky D, Vogelstein B and Harris CC: p53 mutations in human cancers. Science 253: 49-53, 1991

24. Brugarolas J, Chandrasekaran C, Gordon JI, Beach D, Jacks T and Hannon GJ: Radiation-induced cell cycle arrest compromised by p21 deficiency. Nature 377: 552-557, 1995.

25. Soussi T and Béroud C: Assessing TP53 status in human tumours to evaluate clinical outcome. Nat Rev Cancer 1: 233-240, 2001.

26. Sturm I, Bosanquet AG, Hermann S, Guner D, Dörken B and Daniel PT: Mutation of p53 and consecutive selective drug resistance in B-CLL occurs as a consequence of prior DNA-damaging chemotherapy. Cell Death Differ 10: 477-484, 2003.

27. Gavrilov V, Leibovich Y, Ariad S, Lavrenkov K and Shany S: A combined pretreatment of 1,25-dihydroxyvitamin D3 and sodium valproate enhances the damaging effect of ionizing radiation on prostate cancer cells. J Steroid Biochem Mol Biol 121: 391-394, 2010.

28. Gan CP, Hamid S, Hor SY, Zain RB, Ismail SM, Wan Mustafa WM, Teo SH, Saunders N and Cheong SC: Valproic acid: Growth inhibition of head and neck cancer by induction of terminal differentiation and senescence. Head Neck 34: 344-353, 2012.

29. Li XN, Shu Q, Su JM, Perlaky L, Blaney SM and Lau CC: Valproic acid induces growth arrest, apoptosis, and senescence in medulloblastomas by increasing histone hyperacetylation and regulating expression of p21Cip1, CDK4 and CMYC. Mol Cancer Ther 4: 1912-1922, 2005.

30. Gire V, Roux P, Wynford-Thomas D, Brondello JM and Dulic V: DNA damage checkpoint kinase Chk2 triggers replicative senescence. EMBO J 23: 2554-2563, 2004.

31. Aliouat-Denis CM, Dendouga N, Van den Wyngaert I, Goehlmann H, Steller U, van de Weyer I, Van Slycken N, Andries L, Kass S, Luyten W, et al: p53-independent regulation of p21Waf1/Cip1 expression and senescence by Chk2. Mol Cancer Res 3: 627-634, 2005. 